\title{
Introduction to immunology at The University of Iowa
}

\author{
Gail A. Bishop
}

Published online: 24 May 2014

(c) Springer Science+Business Media New York 2014

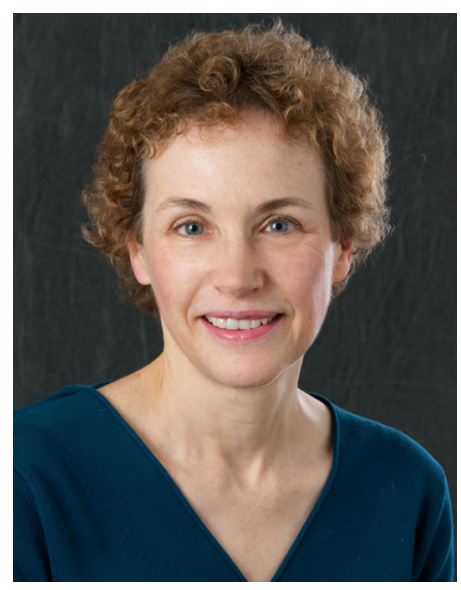

Gail A. Bishop

A previous issue of Immunologic Research featured a short history and description of our immunology community in 2007 (Bishop, G. A. Immunol. Res. 2007, 39:1-3). Where are we now, 7 years later? Our Interdisciplinary Graduate Program in Immunology (http://immuno.grad.uiowa.edu/) continues as an active focus of training for $\mathrm{PhD}$ and MD$\mathrm{PhD}$ students and is now in its 21 st year, and its 4 th cycle of NIH T32 support. After having the honor of directing this program for 15 years, in 2013, I was pleased to welcome Dr. Steven Varga as my successor. A new initiative has been launched by the Carver College of Medicine, the University of Iowa Center for Immunology and ImmuneBased Diseases, http://www.medicine.uiowa.edu/immunol ogy/. This Center was established to achieve an integrated and multidisciplinary approach to the study of immunology in its diverse manifestations and thus facilitate collaboration and communication between faculty, fellows, students and staff at all levels across campus. The Center works closely with the Graduate Program in Immunology, but also includes many immunologists who are not directly involved in graduate training. In this regard, we also interact closely with other Centers on campus, including the Holden Comprehensive Cancer Center and the Fraternal Order of Eagles Diabetes Research Center, both of which sponsor joint activities with the Center for

G. A. Bishop ( $\square)$

The University of Iowa, 2193B MERF, 375 Newton Rd., Iowa City, IA 52242, USA

e-mail: gail-bishop@uiowa.edu

G. A. Bishop

Iowa City VAMC, Iowa City, IA 52242, USA
Immunology. Key activities of our Center include an annual research Retreat and a monthly Research-in-Progress series.

The ongoing research projects of immunologists at The University of Iowa continue to cover a wide variety of topics, and investigators have applied their areas of expertise to new and emerging problems relevant to human and animal health. An important focus here has long been host responses to infectious pathogens. These include viral, bacterial and parasitic organisms. In this issue, Drs. Legge, Waldschmidt and colleagues discuss features of both lymphocytes ( $\mathrm{T}$ and $\mathrm{B}$ ) and dendritic cell responses to influenza virus, and Dr. Butler reviews an important respiratory virus of the porcine population. Key features of the $\mathrm{CD} 4^{+} \mathrm{T}$ cell response to another important respiratory virus, RSV, are reviewed by Dr. Varga and members of his laboratory. Another class of respiratory viruses of great current interest and relevance, coronaviruses, is discussed in the context of the host $\mathrm{T}$ cell response by Dr. Perlman and colleagues.

Several investigators at The University of Iowa focus upon aspects of the mammalian host response to the tropical parasite Leishmania. As global climate steadily warms, such pathogens emerge in larger proportions of the world, and become of increasing public health importance. This issue includes reviews of the roles of eosinophils and mast cells in this host response (Drs. Rodriguez and Wilson), host responses mediated through the innate immune NLR proteins in parasitic diseases (Drs. Clay, Sutterwala and Wilson) and how members of the MAP kinase family influence chronic Leishmania infections (Drs. Martinez and Petersen). Another parasitic infection of major global health impact is malaria. Dr. Harty and Katherine Doll review features of the host response relevant to vaccination against this organism. 
Several University of Iowa investigators are pursuing a better understanding of host-microbe interactions with Staphylococcus bacteria, important human pathogens involved in many types of serious infections. Reviews focusing on mechanisms of pathology in these host-pathogen interactions are provided by Drs. Schlievert and Salgado-Pabon and their colleagues.

Another major focus of University of Iowa immunology continues to be the interaction between the host immune response and various types of malignancies. This includes both studies of cancers of immune cells themselves, as well as the study of host-tumor interactions, and how to exploit the host immune response to fight malignant diseases. Several articles in this issue highlight some of the ongoing work here in both these areas. Drs. Janz, Zhan, Tricot and their colleagues discuss the promise of targeting IL-6 as part of new therapeutic strategies in the B lymphocyte malignancy, multiple myeloma. Another type of B cell tumor, B cell lymphoma, is the focus of a review by Dr. Weiner and colleagues, who discuss mechanisms of efficacy in monoclonal antibody-based therapies. A number of immunologists here also pursue various immune-based therapies for solid tumors, including melanoma and various types of carcinoma. Examples of this work are provided in the review by Drs. Chehval and Norian, who discuss the timely topic of the impact of obesity upon host responses to renal cell carcinoma. Dr. Salem and colleagues discuss their work using biodegradable particles in cancer vaccines. Additionally, Dr. Lubaroff has brought from bench to bedside a longstanding project to develop a prostate cancer vaccine. This successful work is discussed in two reviews here, by Lubaroff et al. and Uhlman et al.

It has become increasingly appreciated that chronic inflammation makes critical contributions to the pathogenesis of many human diseases. Immunologists at The University of Iowa study this process in a variety of inflammatory and autoimmune conditions. Featured here are reviews of work on immune system contributions to hypertension by Dr. Abboud and colleagues, as well as to multiple sclerosis, by Dr. Karandikar and colleagues. Dr. Hirsch and his laboratory review their work on follistatinlike protein 1 and its potential roles in chronic inflammatory diseases such as rheumatoid arthritis, while Drs. Messingham, Holahan and Fairley provide a prospective on the role of $\operatorname{IgE}$ autoantibodies in an inflammatory skin disease, bullous pemphigoid. Continuing on the theme of skin cells, Dr. Zavazava and colleagues review their recent efforts to manipulate dermal fibroblasts to become pancreatic beta cells, for use in therapies for diabetes.

Of course, underlying all work that applies principles of immunity to disease are basic studies of how immune cells develop, function and interact. Such work has been pursued by many of our immunologists for decades and has formed an irreplaceable basis for subsequent translational studies. Reviews on such topics are also included in this issue. Drs. Gioannini, Weiss and colleagues review their longstanding research program that has revealed important aspects of the function of the major innate immune receptor TLR4. Although TLRs have been most often studied on myeloid cells, where they play crucial roles in innate immunity, they are also expressed by lymphocytes. B lymphocytes, in particular, express many TLRs and are highly responsive to their signals, a topic reviewed by Drs. Buchta and Bishop.

A major longstanding research interest at The University of Iowa is understanding the details of $\mathrm{T}$ lymphocyte function and regulation, and several aspects of this topic are reviewed here. Farrah Steinke and Dr. Xue review their recent studies of the roles of Tcf1 and Lef1 transcriptional regulators in $\mathrm{T}$ cell development and lineage determination. Reviews that focus upon functions and consequences of $\mathrm{T}$ cell antigen receptor roles are provided by Matthew Martin and Dr. Badovinac and Drs. Chapman and Houtman. A topic of considerable recent interest in the cancer immunotherapy field is molecules that mediate $\mathrm{T}$ cell suppression, or 'checkpoint blockade.' Basic studies on the function of one of these molecules, Tim-3, are reviewed by Drs. Gorman and Colgan.

The reviews included here provide an overview of the variety of topics, both basic and disease-oriented, that are studied by immunologists at The University of Iowa. However, each of the laboratories that participated in this special issue has additional ongoing projects, and both new colleagues and new research directions regularly join our community. Thus, the reader is encouraged to visit our websites mentioned above, and is also encouraged to visit us here-we would be delighted to welcome you to Iowa City, and to discuss opportunities here in research and training! 